\title{
Revenue-driven Lightpaths Provisioning over Optical WDM Networks Using Bee Colony Optimization
}

\author{
Goran Z. Marković \\ University of Belgrade - Faculty of Transport and Traffic Engineering, \\ Vojvode Stepe 305, \\ Belgrade, 11000, Serbia \\ E-mail: g.markovic@sf.bg.ac.rs
}

Received 6 January 2016

Accepted 5 December 2016

\begin{abstract}
This paper aims to study the lightpaths provisioning problem in optical WDM networks with scarce available wavelengths under the static (off-line) traffic demands such that network operator's (NO's) revenue is maximized. To achieve this goal, a NO has to be addressed with the issue how to solve the call admission control jointly with the lightpaths routing and wavelength assignment (RWA) problem in efficient manner. The improved bee colony optimization (BCOi) metaheuristic is applied to solve the considered revenue maximization (Max-Rev) problem. We evaluated the performances of the proposed BCOi Max-Rev framework by performing numerous simulation experiments in different realistic WDM optical network topologies. We observed that our BCOi Max-Rev algorithm is an efficient tool to produce high quality solutions within reasonable amount of CPU time. It has been proved that BCOi Max-Rev solutions just slightly deviate from optimal solutions (at most 1\%) and considerably outperform some heuristic algorithms, such as the Max-Profit and FCFS. In addition, our Max-Rev BCOi algorithm is able to produce better solution quality compared to the constructive BCO approach (up to $3.5 \%$ in the case of NSFNet and 5\% in the case of EON). Finally, we compared the BCOi to differential evolution (DE) approach in the case of more complex networks, such as the USA optical network topology. The results show that our BCOi always outperforms DE metaheuristic, whereby the profit improvement could reach up to $20 \%$ in some instances.
\end{abstract}

Keywords: bee colony optimization (BCO), lightpath, optical network, routing and wavelength assignment (RWA), revenue maximization.

\section{Introduction}

Optical networks employing wavelength division multiplexing (WDM) and wavelength routing techniques are being considered as a candidate solution for future backbone infrastructures ${ }^{1}$. Such networks are able to provide practically unlimited bandwidths required for a variety of advanced and upcoming telecommunication services and applications. With the purpose to offer high data rates and guaranteed quality of service to end users network operators (NOs) have to provide a number of connections over optical network infrastructure. All-optical connections established between two wavelength routing nodes are called the lightpaths. While establishing the lightpaths a NO tries to maximize its own revenue by charging the occupation of network resources. To achieve this goal NO has to be addressed how to solve the call admission control jointly with the lightpaths routing and wavelength assignment (RWA) problem ${ }^{1}$. Various services over future backbone optical networks, like lambda grids or optical virtual private networks (oVPNs) could be characterized as off-line (or static) traffic demands. In such case, the traffic demands are 
specified in advance before the connections have to be established. By knowing the complete set of demands, a more efficient network resource usage (and consequently more revenues) could be achieved compared to on-line (dynamic) traffic case in which future demands are not known a priori.

So far, various approaches to solve the revenue (profit) maximization problem in optical WDM networks have been proposed in literature ${ }^{2-6}$. In Ref. 2, the problem of maximizing profits is mathematically formulated as an integer linear programming (ILP) problem. Since the optimal provisioning of lightpaths is NP-hard combinatorial problem, several heuristic algorithms are proposed to solve the problem assuming both an off-line and an on-line traffic model. The authors of Ref. 3 proposed an efficient near-optimal algorithm based on Lagrangean relaxation (LGR) approach to solve the lightpath provisioning problem in which the revenue from accepting call requests is to be maximized. In Ref. 4 a polynomial-time algorithm to design a $k$-colourable set of paths in a chain WDM topology as well as 2-approximation algorithm for ring topology are proposed, such that the total profit of the set of designed paths is maximized. In Ref. 5, the authors propose a heuristic algorithm based on the genetic algorithm (GA) approach for addressing the problem of optimal provisioning of virtual network requests with unsplittable and splittable flows in cloudbased data centres such that total revenue is maximized. Following recent advancing in optical technology, the authors of Ref. 6 proposed two wavelength assignment approaches for flex-grid optical path topology, with the goal to maximize the total profit.

In this paper, the bee colony optimization (BCO) metaheuristic is applied to solve the revenue maximization (or shortly Max-Rev) problem in optical WDM networks. Various studies proved that $\mathrm{BCO}$ is a fast, robust and efficient optimization tool, which can produce high quality solutions together with small computational complexity $^{7-15}$. A comprehensive overview of $\mathrm{BCO}$ metaheuristic with its various applications could be found in Refs. 10 - 11. Inspired by a number of successful previous $\mathrm{BCO}$ implementations, our goal here is to demonstrate the quality and efficiency of the BCO approach while solving the considered Max-Rev problem. To the best of our knowledge, this study is the first application of the $\mathrm{BCO}$ approach to solve the revenue optimization problem in communication networks. Previous results have proved that $\mathrm{BCO}$ could be better-quality approach compared to other heuristic and metaheuristic algorithms. It indicates that $\mathrm{BCO}$ could be a promising candidate to solve various complex optimization problems in WDM optical networking, such as the considered Max-Rev problem.

The main contributions of this paper are following:

i) We adapted the $\mathrm{BCO}$ model previously proposed in Ref. 9 to solve the considered Max-Rev problem in optical WDM networks. Although the objective in Ref. 9 is to maximize the number of established lightpaths, it does not consider the economic aspects, which are highly important from network operator's point of view. However, it is obvious that maximizing only the number of established lightpaths does not necessarily imply the maximal NO's revenues, too. The exception is the scenario where all the lightpaths are equally charged, which is not too realistic case. Hence, the optimization task is to make a decision what is the best set of lightpath demands to be established together with their routes and wavelengths, such that the total NO's revenue is maximized under the limited available wavelengths in a given WDM network.

ii) $\mathrm{We}$ developed an entirely new Max-Rev optimization model by implementing the improvement $\mathrm{BCO}$ approach (shortly, BCOi). BCOi is a more recent approach that is firstly proposed in Ref. 12 to solve the well-known $p$-centre problem in graph theory. It will be shown that our Max-Rev BCOi algorithm is able to produce near-optimal (or even optimal) solutions, which are superior compared to the constructive version of the BCO-model $^{9}$ as well as to some simple heuristics, such as the Max-Profit and first-come-firstserved (FCFS) algorithms proposed in Ref. 2.

iii) In addition, we have applied the differential evolution (DE) metaheuristic to the considered MaxRev problem in order to perform a more reasonable comparison of BCOi to other similar optimization approaches. DE is a population based random search technique, which performs iteratively through a number of generations to find the best solution to the problem. Like the GA approach, DE also uses evolutionary operators (mutation, recombination and selection) to modify the population of individuals (set of candidate solutions) in each generation. The main control parameters of $\mathrm{DE}$ are: the mutation constant $(M)$, which controls the mutation strength; the recombination 
constant $(R C)$, which increases the diversity in the mutation process; and the population size $(N P)$ that is an integer that depends on the dimension of the problem. ${ }^{16-19}$ DE searching procedure starts with a randomly created initial population and iterates by creating new populations until a given stopping criterion is satisfied. A more comprehensive theoretical description of the DE approach could be found in Ref. 16. Several studies in optical networking show that DE outperforms some other evolutionary algorithms ${ }^{17-19}$. However, it has been recently shown in Ref. 20 that $\mathrm{BCO}$ is superior compared to DE as well as to particle swarm optimization (PSO) metaheuristics in terms of the solution quality and the efficiency while solving the wavelength converters placement problem in WDM networks. This encouraged us to choose the BCOi as an efficient tool to solve the Max-Rev problem in this paper.

The paper is organized as follows. In Section 2, the statement of the Max-Rev problem is given. The main principles of the BCOi metaheuristic are described in Section 3. The proposed BCOi model for solving the considered Max-Rev problem is introduced in Section 4. In Section 5, we demonstrate the numerical results of performance study and comparisons under various WDM optical network topologies. Finally, some conclusions are given in the last section.

\section{Max-Rev Problem Statement}

We consider an optical WDM physical network topology that consists of set of network nodes $N$ (equipped with optical cross-connects), which are interconnected by set of optical links $L$ in an arbitrary (mesh) topology. We make the following assumptions: every link in a physical optical network consists of a pair of optical fibres; each fibre has the same set of available wavelengths $W$; wavelength converters are not available at network nodes, hence the wavelength continuity constraint has to be satisfied; there is no limitation on the number of optical transceivers at network nodes; each lightpath is exclusively used for one source-destination node pair $(s, d)$; the set of requested lightpath demands $K$ as well as the set of candidate shortest paths $P_{k}$ for each lightpath demand are given in advance. Each lightpath demand is specified by its starting and ending time during a (short) planning horizon. Such situation could be usually found in practice when there is no need to establish the lightpaths for an infinite duration, but rather for a particular period during a day (for example several hours) or for some other periods. We assumed also that every lightpath demand contributes to different NO's revenue. The revenue for a lightpath could depend on various aspects, such as for example the geographical distance between wavelength routing nodes, duration of a lightpath, time periods during a planning horizon, requested priority of a lightpath demand, quality of service parameters etc. For simplicity, we assume here that revenue for a lightpath depends on its duration and the period during a planning horizon (for example, NO could offer lower prices during the periods of light traffic demands and higher prices during busy hours). Hence, we define the revenue $R_{k}$ for establishing a lightpath demand $k$ as follows:

$$
R_{k}=\sum_{d=1}^{D_{k}} r_{d} \quad \forall k=1,2, \ldots,|K|
$$

where:

$D_{k}$ - duration of a lightpath demand $k$, which is multiple of time slot units (every time slot unit is of duration $\Delta T$ ) $d$ - index of time slot unit $\Delta T$ during a planning horizon,

$r_{d}$ - revenue per time slot $d$,

$k$ - index of a lightpath demand,

$|K|$ - total number of lightpath demands.

Under the above assumptions, the considered MaxRev problem could be expressed as follows: for given optical WDM physical network topology, traffic demands and number of wavelengths on WDM links, the objective is to determine the best (optimal) set of lightpaths to be established jointly with their routes and assigned wavelengths such that total NO's revenue is maximized. To find the optimal solution of the considered problem, we used the integer linear programming (ILP) formulation proposed in Ref. 3. The rest notations used in this formulation are following:

constants:

$\delta_{p l}=\left\{\begin{array}{l}1, \text { if path } p \in P_{k} \text { includes link } l \in L, \\ 0, \text { otherwise }\end{array}\right.$

$T$ - set of time events (for detail insight of time events see Ref. 3), 
$\sigma_{k t}=\left\{\begin{array}{l}1, \text { if lightpatk } k \in K \text { includes event time } t \in T, \\ 0, \text { otherwise }\end{array}\right.$

binary variables:

$x_{p w}^{k}=\left\{\begin{array}{l}1, \text { if a lightpath } k \in K \text { traverse path } p \in P_{k} \\ \quad \text { using the wavelength } w \in W, \\ 0, \text { otherwise }\end{array}\right.$

$y_{k}=\left\{\begin{array}{l}1, \text { if a lightpath } k \in K \text { is established } \\ 0, \text { otherwise }\end{array}\right.$

Following is the mathematical (ILP) formulation of the considered Max-Rev problem ${ }^{3}$ :

$$
\max \sum_{k \in K} R_{k} y_{k}
$$

subject to:

$$
\begin{gathered}
\sum_{p \in P_{k}} \sum_{w \in W} x_{p w}^{k}=y_{k}, \forall k \in K \\
\sum_{p \in P_{k}} \sum_{w \in W} x_{p w}^{k} \leq 1, \forall k \in K \\
\sum_{k \in K} \sum_{p \in P_{k}} x_{p w}^{k} \delta_{p l} \sigma_{k t} \leq 1, \quad \forall w \in W, l \in L, t \in T \\
x_{p w}^{k}=0 \text { or } 1, \forall p \in P_{k}, w \in W, k \in K \\
y_{k}=0 \text { or } 1, \forall k \in K
\end{gathered}
$$

The objective function, given by Eq. (2) is to maximize the total revenue for a NO. It could be noticed that in a special case, when all lightpath demands have the same revenue $R_{k}=1$, the considered ILP formulation is reduced to the problem of maximization the number of established lightpaths. The constraints (3) to (4) ensure that mostly one lightpath should be established for each traffic demand. Constraints (5) prevent wavelength conflicts, i.e. ensure that on every link only one wavelength (lightpath) could be used at any time slot. Constraints (6) and (7) ensure that corresponding binary variables could take only one value ( 0 or 1$)$. A binary value of variable in constraints (7) determines if the lightpath demand is established $\left(y_{k}=1\right)$ or not $\left(y_{k}=0\right)$. The ILP formulation is shown to be NP-hard optimization problem that could be solved exactly only in case of small problem dimensionality. However, ILP becomes computationally intractable even for moderately large networks or incurs a large computational time to find the optimal solution. Hence, heuristic based approaches should be used to find the (suboptimal) solution. We used here the BCOi metaheuristic approach to solve the considered optimization problem in case of realistic size optical WDM networks. Before presenting the BCOi implementation for considered Max-Rev problem, we present shortly the main principles of BCO metaheuristic in following section.

\section{BCO Main Principles}

BCO metaheuristic is a population based stochastic random-search technique that is inspired by the foraging habits of natural bees looking for nectar sources ${ }^{8-15}$. It is originally proposed by Teodorović and Lučić in Ref. 14.

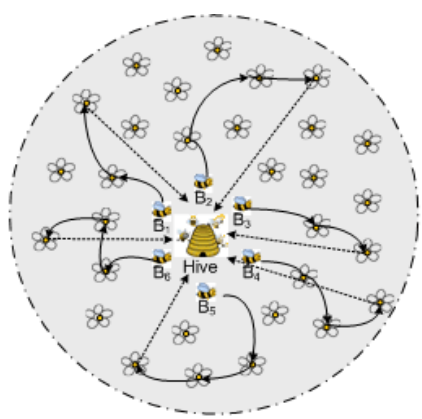

(a) First forward \& backward pass ( $S=1)$

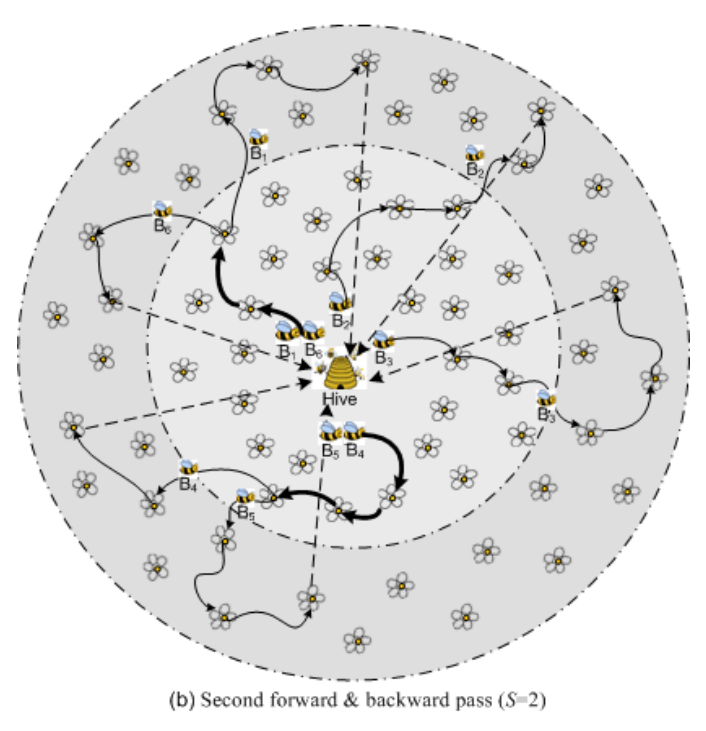

Fig. 1. Illustration of: a) the first $(S=1)$ and (b) the second $(S=2)$ forward and backward passes

Similar to the foraging habits of natural bees, the artificial bees in BCO perform the examination of the search space looking for the best solutions. Each bee in a population generates one solution to the considered optimization problem. $^{7-15}$ 


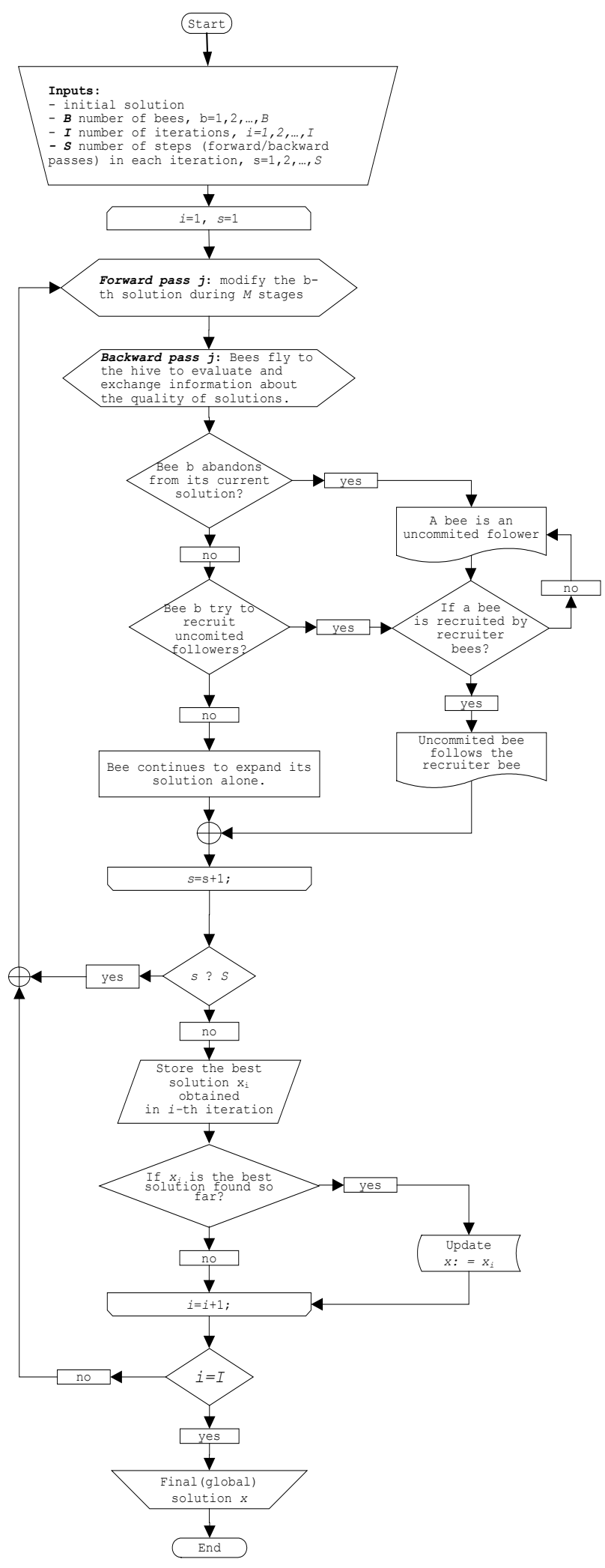

Fig. 2. The flowchart of the BCO algorithm
During the iterative searching procedure, bees generate (or improve) its individual solutions yielding to new ones. In order to find the best solution, artificial bees perform cooperative decisions in the hive by exchanging the information about the quality of their current solutions. In this way, bees progressively discover solutions that are more promising and gradually leave worse ones. There are two approaches of the BCO metaheuristic ${ }^{10,11}$ : the constructive BCO and the improvement (BCOi) versions. The first approach is based on constructive steps in which bees begin to search for the best solution from an empty solution and incrementally expand their solutions during the search process. In the improvement (BCOi) approach bees start with a complete initial solution of the considered problem and modify it through the search process in order to obtain the best possible final solution. We used the second (BCOi) approach in this study.

As it is already mentioned, the searching procedure in BCO algorithm is performed iteratively ${ }^{10,11}$. Each iteration contains a number of steps $(S)$ and each step consists of the so called forward pass $(F P)$ and backward pass $(B P)$, which are performed alternatively. The number of forward/backward passes (or steps $S$ ) is specified in advance. In each forward pass, every bee makes a number of modifications $(M)$ of its current solution. At the beginning of search process, all artificial bees are placed in the hive. The number of bees $(B)$ engaged in the search process is specified in advance, too. An illustration of the first forward and backward pass is shown in Fig.1 (a). To simplify the illustration, we assumed that there are $B=6$ bees in the hive. At the beginning of search process bees start the searching from an initial complete solution that could be generated randomly or by applying some heuristic algorithm. By knowing the initial solution, all bees start to fly through the search space and modify the current solution trying to improve it in each step. During a forward pass every bee flies through several stages (which are represented by flowers in Fig. 1).

In each stage, every bee modifies its current solution compared to the previous stage yielding to a new solution. ${ }^{10-13}$ The number of solution modifications $(M)$ within each forward pass $(F P)$ could be specified in advance or randomly chosen at the beginning of each forward pass. After $M$ modifications of solutions performed by all bees, the first forward pass is finished 
and the second phase of the BCO step, the so-called backward pass $(B P)$ begins. During the $B P$, all bees fly back to the hive (it is illustrated by straight dash lines in Fig.1) in order to share information and compare the quality of generated solutions. The quality of bee's solution corresponds to the considered objective function value. It is assumed that each bee in the hive could be aware of its own solution quality and at the same time, she can obtain the information about solutions' quality of all other bees. By exchanging the information about the quality of individual solutions, every bee has to decide whether it will be loyal to its individual solution or not ${ }^{8}$. Such decision is made with a certain probability such that the bees with better solution quality (objective function value) have more chances to keep their solutions. The bees that are loyal to their solutions create the set of recruiter bees, while the other bees from colony that give up from their solutions become uncommitted followers. Recruiter bees advertise their solutions in the hive in such way trying to attract some of the uncommitted followers to join them during a recruitment process. Each uncommitted follower has to decide whether in the next step (forward pass) it will continue to explore its own solution, or to start jointly exploring one of the solutions being advertised by the recruiter bees. This decision is taken with a certain probability such that better advertised solutions have greater chance to be chosen by followers for further exploration ${ }^{10,11,15}$.

The set of recruiter bees and uncommitted followers are changing from one backward pass to another. For example, let assume that after comparing all generated solutions, bees $B_{4}$ and $B_{6}$ decided to abandon from their solutions. Let us also assume that during the recruitment process bee $B_{4}$ decided to join to recruiter bees $B_{5}$ and bee $B_{6}$ decided to join to bee $B_{1}$. When the first backward pass is finished, in the second forward pass $(S=2)$ bee $B_{4}$ will fly through the search space along the same path already generated by the bee $B_{5}$. It means that the bee $B_{4}$ will take the same solution as the bee $B_{5}$. In the same way, $B_{6}$ will take (follow) the solution generated by bee $B_{1}$. The recruiter bees $B_{2}$ bee $B_{3}$ continue to fly alone and keep their already generated solutions.

At the end of first pass, all bees are free to make their next individual decisions and to continue to explore the search space during the second forward pass independently, as it is illustrated in Fig.1.(b).
After that, they perform again the backward pass and return to the hive. In the hive bees makes again the same decisions and perform the third forward pass, etc. After $S$ passes are performed, iteration is finished. At the end of iteration, $B$ solutions are obtained. The best found solution during iteration is saved and then the second iteration starts. The $\mathrm{BCO}$ algorithm runs in iterations until a given stopping requirement is satisfied, such as for example the maximum number of iterations $(I)$ or the number of iterations without the improvement of the objective function value, etc ${ }^{7,10}$. At the end of iterative process, the best found solution (socalled global best) is reported as the final one. Fig 2 shows the flowchart of the BCOi algorithm.

\section{BCOi Implementation for Max-Rev Problem}

In this section, we present the implementation of described BCOi approach to solve the revenue maximization problem in optical WDM networks. The proposed model contains the following steps:

\subsection{Initial solution}

As it has been mentioned, an initial complete solution has to be generated before the $\mathrm{BCO}$ iterative searching procedure begins. As an initial solution for our model, we used the final solution generated by the $\mathrm{BCO}$ algorithm proposed in Ref. 9 that is based on constructive approach. In such a way, we are to create an integral optimization model in which the first phase is based on the constructive BCO approach and the second phase is an improvement (post-optimization) procedure performed by BCOi approach. The initial solution could be also generated using some other heuristics, such as the Max-Profit and FCFS algorithms ${ }^{2}$. In Max-Profit algorithm, the lightpath demands are sorted in the ordered list according to the prospective profit (revenue) value, beginning with the lightpath demand with maximal profit at the top of the list. After that, one by one lightpath demand from the list is tried to be established. In the case of FCFS heuristic, the lightpath demands are sorted in order of their time of arrival in a network (like in the case of online traffic scenario). Although very simple, it has been shown that Max-Profit heuristic is able to produce in many instances near optimal solutions ${ }^{2}$. However, it will be shown that there is still a possibility to improve such solutions using our BCOi Max-Rev approach. 


\subsection{Changing of solutions}

During the search process, all bees make the modifications of their solutions throughout $S$ forward passes within iteration. In each forward pass $(F P)$, every bee changes its solutions by making $M$ modifications to the previous solution. ${ }^{10-12}$ In our model, each bee selects $M$ established lightpaths to be released from current solution. The number of released lightpaths in each forward pass is chosen randomly, but it is limited to be at most the pre-specified percent of total number of established lightpaths. In such a way, $M$ lightpaths are removed from the current solution and new lightpaths are tried to be established in a random order (from the set of removed lightpaths together with previously unsatisfied lightpath demands). To establish a lightpath, the RWA problem has to be solved. For the routing sub-problem, we used the set of pre-determined $k$-shortest paths for every node pair $(s, d)$. Then, the first feasible route (with at least one same free wavelength on every link on the route) is chosen. The first-fit (FF) strategy is used for wavelength assignment sub-problem. If a feasible RWA solution exists, a lightpath is established and the objective function value is updated.

\subsection{Comparing the solutions}

After modification of solutions, all artificial bees are flying back to the hive to evaluate and compare the quality of their individual solutions..$^{7-14}$ The comparison of solutions is based on objective function value (the total NO's revenue) that has to be maximized. In order to decide which bee will be loyal to its previously found solution, we use the probability $p_{b}^{s+1}$ that $b$-th bee, at the beginning of the new forward pass $s+1$, will retain its solution as follows ${ }^{10,11}$ :

$$
p_{b}^{s+1}=e^{-\frac{O_{\max }-O_{b}}{s}}, b=1,2, \ldots B
$$

where:

$O_{b}$ - denotes the normalized objective function value of the solution created by the $b$-th bee;

$O_{\max }$ - represents maximum over all normalized values of solutions to be compared;

$s$ - the ordinary number of step $(s=1,2, \ldots, S)$.
The normalized value of the objective function $O_{b}$ for solution created by the $b$-th bee could be determined as ${ }^{10,11}$ :

$$
O_{b}=\frac{F_{b}-F_{\min }}{F_{\max }-F_{\min }}, \quad b=1,2, \ldots, B
$$

where $F_{\min }$ and $F_{\max }$ are minimal and maximal values of the objective function $F$ obtained by all bees, respectively, and $F_{b}$ is the objective function value obtained by the $b$-th bee. From Eq. (9), it could be observed that if the revenue value $F_{b}$ obtained by the $b$ th bee is closer to the worst value of all obtained solutions $F_{\min }$ then its normalized value $O_{b}$ is smaller and vice versa. Based on Eq. (8), a lower $O_{b}$ value will decrease the probability that bee $b$ will be loyal to its previous solution. ${ }^{10}$ In other words, the greater the revenue value $F_{b}$ obtained by the bee $b$, the greater is the chance that bee $b$ will keep further its solution. Additionally, the greater the ordinary number of the forward pass $s$, the higher the influence of the already discovered solution will be. Based on Eq. (8) and randomly generated number rand, each artificial bee decides whether to be uncommitted follower or to continue with exploring its own solution. If rand $\leq p_{b}^{s+1}$ the bee stays loyal to its own solution. Otherwise, the bee $b$ becomes an uncommitted follower ${ }^{7-15}$.

\subsection{Recruitment procedure}

After individual bees' solutions are compared, the bees are disjoined into two sets: the recruiter bees $R$ and the rest $B-R$ bees are the uncommitted bees. ${ }^{7-9}$ Assume that the bee $b$ decides at the beginning of new forward pass to drop out its solution and to follow some of the recruiter bees. Within the recruiting process, an uncommitted bee decides what recruiter bee it will follow, taking into account the quality of all advertised solutions. The probability that $b$ 's solution will be chosen by any uncommitted bee could be defined as follows ${ }^{10,11}$ :

$$
p_{b}=\frac{O_{b}}{\sum_{k=1}^{R} O_{k}}, b=1,2, \ldots, R
$$

where:

$O_{k}$ - represents the normalized value of the objective function of the $k$-th advertised solution, and

$R$ - denotes the number of recruiter bees. 
Using the Eq. (10) and a random number generator, each uncommitted follower joins one recruiter bee based on roulette wheel method ${ }^{10,11}$. After that, uncommitted bees fly together with their recruiters during the next forward pass along the same path as previously discovered by the recruiter bee. At the end of this path, all bees are free to search the solution space (perform next solutions modifications) fully independently. It can be seen from Eq. (9) that if a bee $b$ has found the best solution in step $s$, i.e. solution with maximal revenue value $\left(F_{b}=F_{\max } \Rightarrow O_{b}=O_{\max }=1\right)$, then that bee will fly in the next forward pass $s+1$ along the same path with the probability $p_{b}^{s+1}=1$ (see Eq. 8$) .^{10}$ The higher the $b$-th objective function value $F_{b}$, the greater the probability of flying again along the same path. The described procedure iterates through the prespecified number of iterations $I^{10,11}$. The best solution obtained throughout all iterations is a final (global best) solution of the considered Max-Rev problem.

\section{Experimental Evaluations}

We evaluated the performances of the described MaxRev BCOi framework by performing a numerous simulation experiments. The simulation parameters and the obtained numerical results are given below.

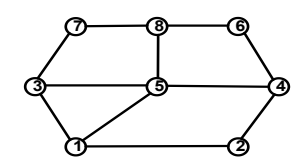

(a)
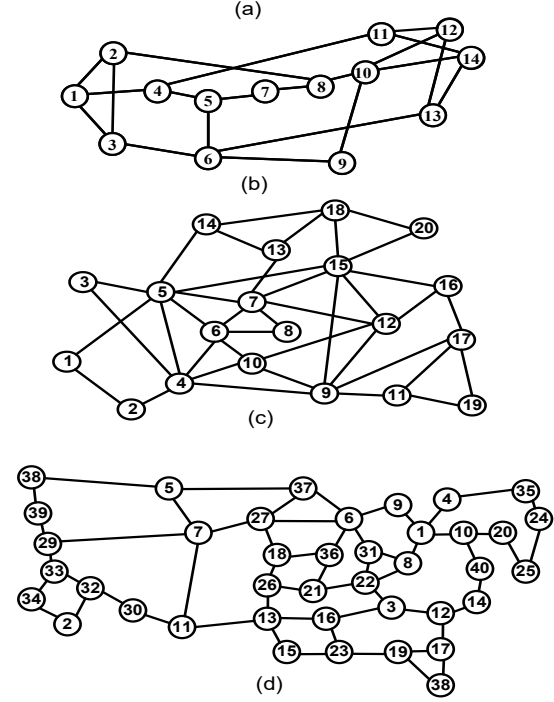

Fig. 3. Considered physical network topologies: (a) small network ( $N=8$ nodes), (b) NSFNet, (c) EON, (d) USA network

\subsection{Simulation parameters}

We have carried out a number of simulation experiments in four optical WDM network topologies shown by Fig.3: (a) a small network with $N=8$ nodes and $L=11$ links together with three realistic size network topologies such as the $(b)$ NSFNet (National Science Foundation Network) with $N=14$ nodes $L=21$ links, (c) EON (European Optical Network) with $N=20$ nodes and $L=39$ links and $(d)$ USA network with $N=40$ nodes and $L=58$ links. Each physical link in all network topologies is assumed to be bidirectional, i.e. it consists of two fibers going in opposite directions. We assumed that same number of wavelengths $|W|$ is available on each link in a network as well as up to $P_{k}=3$ precalculated shortest paths for every node pair $(s, d)$.

We performed a huge number of simulation experiments, but due to limited space we present here only a part of the results, as given in Table 1 . For example, in case of small network with $N=8$ nodes there are 50 experiments (30 experiments for 5 tested algorithms with $|W|=6$ different wavelengths in case of $|K|=50$ demands and 20 additional tests for different number of demands $|K| \neq 50$ ).

Table 1. The number of simulation experiments

\begin{tabular}{ll}
\hline Network topology & Number of experiments \\
\hline Small network (Fig 3a) & $\mathbf{5 0}(30$ for $|K|=50$ and 20 for $|K| \neq 50)$ \\
\hline NSFNet & $\mathbf{5 0}(30$ for $|K|=100$ and 20 for $|K| \neq 100)$ \\
\hline EON & $\mathbf{6 0}(40$ for $|K|=300$ and 20 for $|K| \neq 300)$ \\
\hline USA & $\mathbf{8 4}(30$ for $|K|=100$ and 54 for $|K|=500)$ \\
\hline & $\sum=\mathbf{2 4 4}$ experiments \\
\hline
\end{tabular}

In all simulation experiments, we used the traffic model with uniformly distributed lightpath demands between end nodes. The start time and end time of each lightpath demand are randomly generated following the uniform distribution during the planning horizon. We assumed in all simulations the planning horizon of one day with unit time slot of $\Delta T=1 \mathrm{~h}$. The revenue per a unit time slot $\Delta T$, expressed in monetary units (m.u.) is assumed to be time dependent, as shows the Table 2 .

Table 2. Revenue values per time slots

\begin{tabular}{cccccc}
\hline Period of a day $[\mathrm{h}]$ & $00-08$ & $08-12$ & $12-16$ & $16-20$ & $20-24$ \\
\hline $\begin{array}{c}\text { Revenue per time slot } \\
(\Delta T), r_{d}[\text { m.u. }]\end{array}$ & 10 & 20 & 30 & 20 & 10 \\
\hline
\end{tabular}

Further, we assumed the following input parameters of the BCOi algorithm: number of engaged bees is set to $B=10$ (only in few experiments we used the set of 
$B=20$ bees to test the algorithm's run time), number of steps (forward and backward passes) is set to be $S=40$ and the total number of iterations is set to $I=10$. To perform simulations, the programming code implemented in MATLAB language is developed. All simulation tests have been conducted using the PC equipped with $\operatorname{Intel}(\mathrm{R})$ Core(TM) $2.4 \mathrm{GHz}$ processor and installed (RAM) memory of $3 \mathrm{~GB}$.

\subsection{Numerical results}

To evaluate the solution quality of our BCOi Max-Rev algorithm, we compared it with other approaches, such as the heuristics Max-Profit and FCFS ${ }^{2}$. Further, we compare the BCOi approach with the constructive $\mathrm{BCO}$ algorithm previously proposed in Ref. 9. Additionally, the BCOi approach is also compared with the optimal solution obtained by solving the ILP formulation given by Eqs. (2)-(7). Since the solution of ILP formulation is able to obtain only for small size problems, we solved it only for the small network topology with $N=8$ nodes, shown by Fig 3.a. The summarized comparison results for this network topology are shown by Fig.4.

The objective function value (i.e. total NOs revenues) as well as the number of established (rejected) lightpath demands highly depends on the number of available wavelengths in a network. It could be noticed from Fig. 4.a that our BCOi algorithm is able to produce solutions that always outperform MaxProfit, FCFS and constructive BCO algorithms according to the objective function value. We found that the minimal required number of wavelengths to establish all $(|K|=50)$ lightpaths demands using the BCOi is $|W|=6$, that is the same as optimal (ILP) solution. The objective function value for $\mathrm{BCO}$ and optimal solutions are the same in case of $|W|=1$, too. The blocking probability for considered algorithms is shown in Fig 4.b. The relative gap between obtained solutions is illustrated by graph given in Fig. 4.c. It shows in what percentage the solution obtained by any algorithm is worse than optimal (ILP) solution. As it could be seen, the BCOi approach just slightly (between $0 \%$ and $1.1 \%$ ) deviates from optimal solution, while other considered algorithms have significantly greater deviations from optimal solution. The FCFS heuristic produces the worst results due to fact that in this case we have no information about profit value of demands as in the case of Max-profit. Fig 4.d shows the total revenue value obtained for different number of

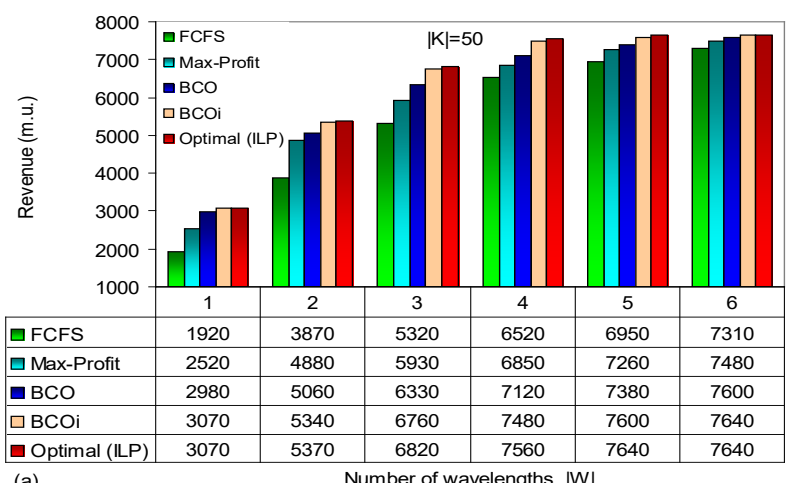

(a) Number of wavelengths, $|\mathrm{W}|$
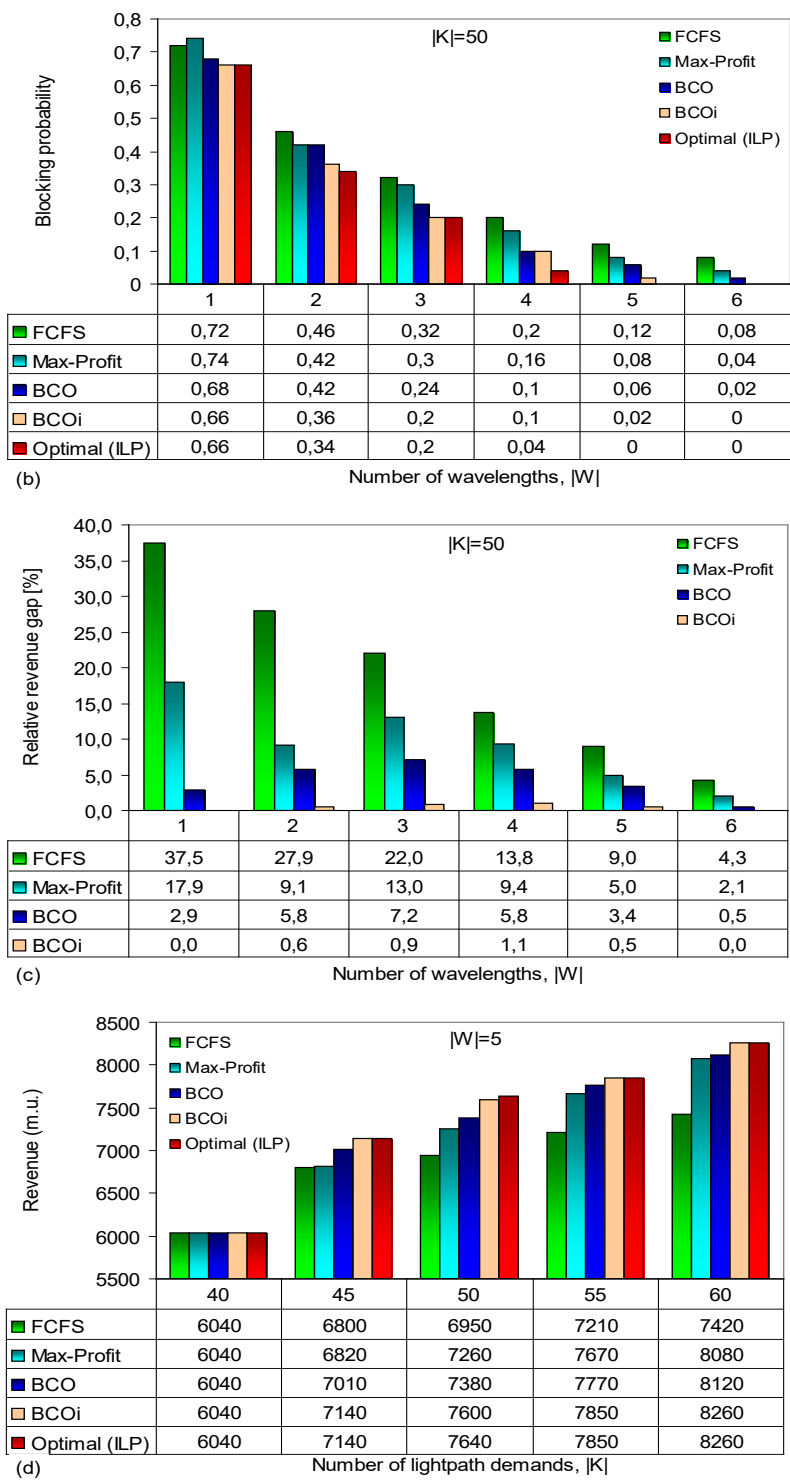

Fig. 4. Performance comparison for small network topology $(N=8)$ : (a) revenue vs. number of wavelengths, (b) number of rejected demands, (c) relative revenue gap, (d) revenue vs. number of demands 

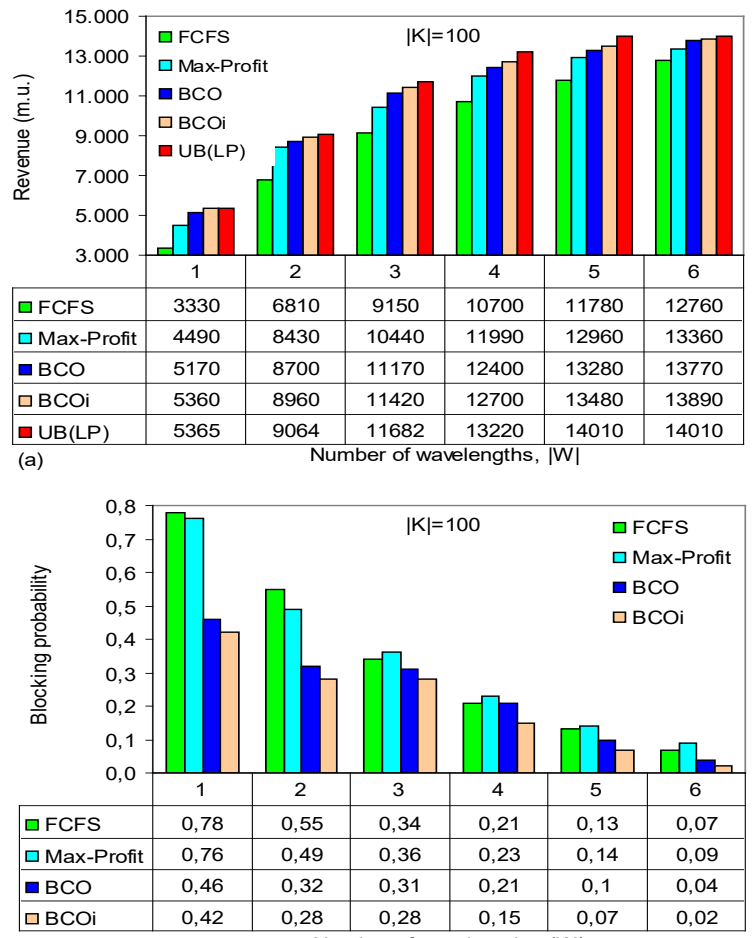

(b) Number of wavelengths, $|\mathrm{W}|$
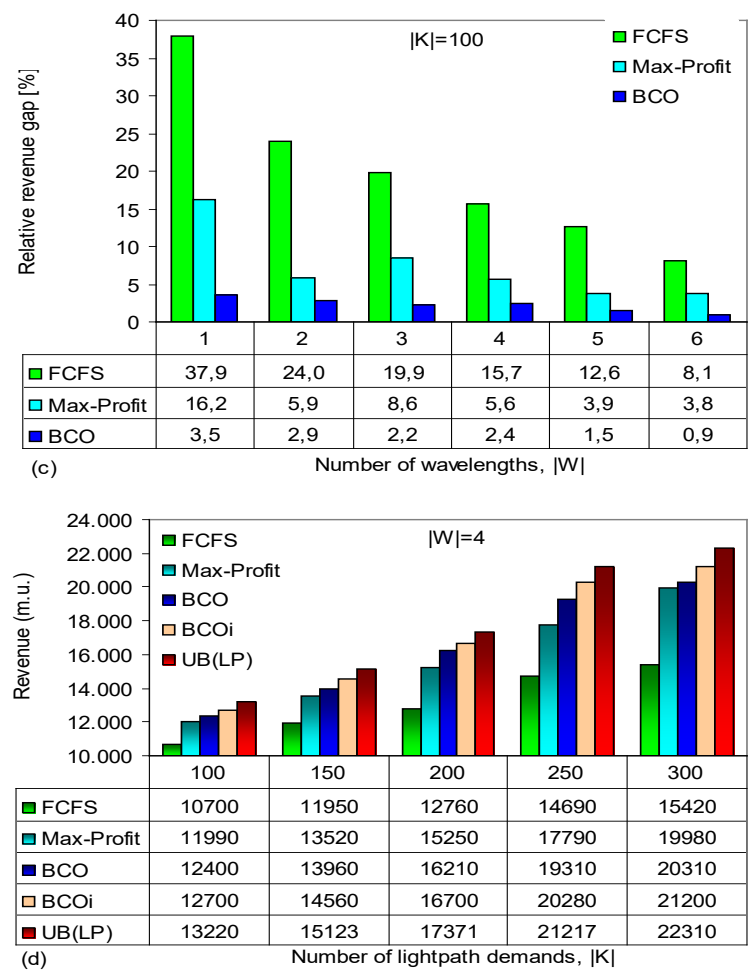

Fig. 5. Performance comparison for NSFNet topology: (a) revenue vs. number of wavelengths, (b) number of rejected demands, (c) relative revenue gap, (d) revenue vs. number of demands lightpaths demands in given network. The optimal (ILP) solution for this network example could be found only for a small number of demands (for $|K| \leq 60$ ).

We have also tested our BCOi algorithm in three realistic size optical networks (NSFNet, EON and USA) with various number of lightpath demands. Figure 5 illustrates the obtained results in the case of NSFNet topology. For this network example, we are not anymore able to find the optimal (ILP) solution, but only the upper bound (UB) values of the LP-relaxation problem, with a reasonable number of lightpath demands $(|K|=100)$. Since the UB values are obtained by relaxation the integer constraints (6) and (7) of ILP model, the resulted solutions are not feasible. In such case, our BCOi model is the only approach that is able to find the feasible solutions of high quality. Once again, the revenue values obtained by our BCOi approach are of superior quality (see Fig 5.a). It could be noticed (from Fig 5.c) that BCOi solution could be better up to $3.5 \%$ compared to the constructive $\mathrm{BCO}$ algorithm. The BCOi solution quality improvements could be significantly higher (up to $16,2 \%$ and 37,9\%) compared to the Max-Profit and FCFS heuristics, respectively. The comparison results in the case of EON network topology are given by Fig. 6. For this network topology, it is not possible to obtain even UB values (except for small number of wavelengths, such as for $|W| \leq 4)$ with a realistic number of lightpath demands $(|K|=300)$. Fig 6 .a shows the reached revenue values of the considered algorithms (for the UB solutions that could not be found, like in the cases of $|W|>4$, a zero value is assigned). The relative solution gap between BCOi approach and other considered algorithms is shown by Fig 6.c. It could be noticed that in this network topology BCOi approach is able to improve the solution quality even up to $5 \%$ compared to the constructive BCO approach. In order to test the possibility of BCOi algorithm to tackle with highly complex problems, we simulated it in the case of greater number of lightpath demands (up to $|\mathrm{K}|=1000$ ). It has been shown that our BCOi approach is able to find high quality solutions within reasonable $\mathrm{CPU}$ times, even in case of such complex problems. The obtained revenue values are compared by Fig. 6.d. As it could be noticed, the BCOi algorithm always outperforms the considered Max-Profit, FCFS and constructive $\mathrm{BCO}$ algorithms. 

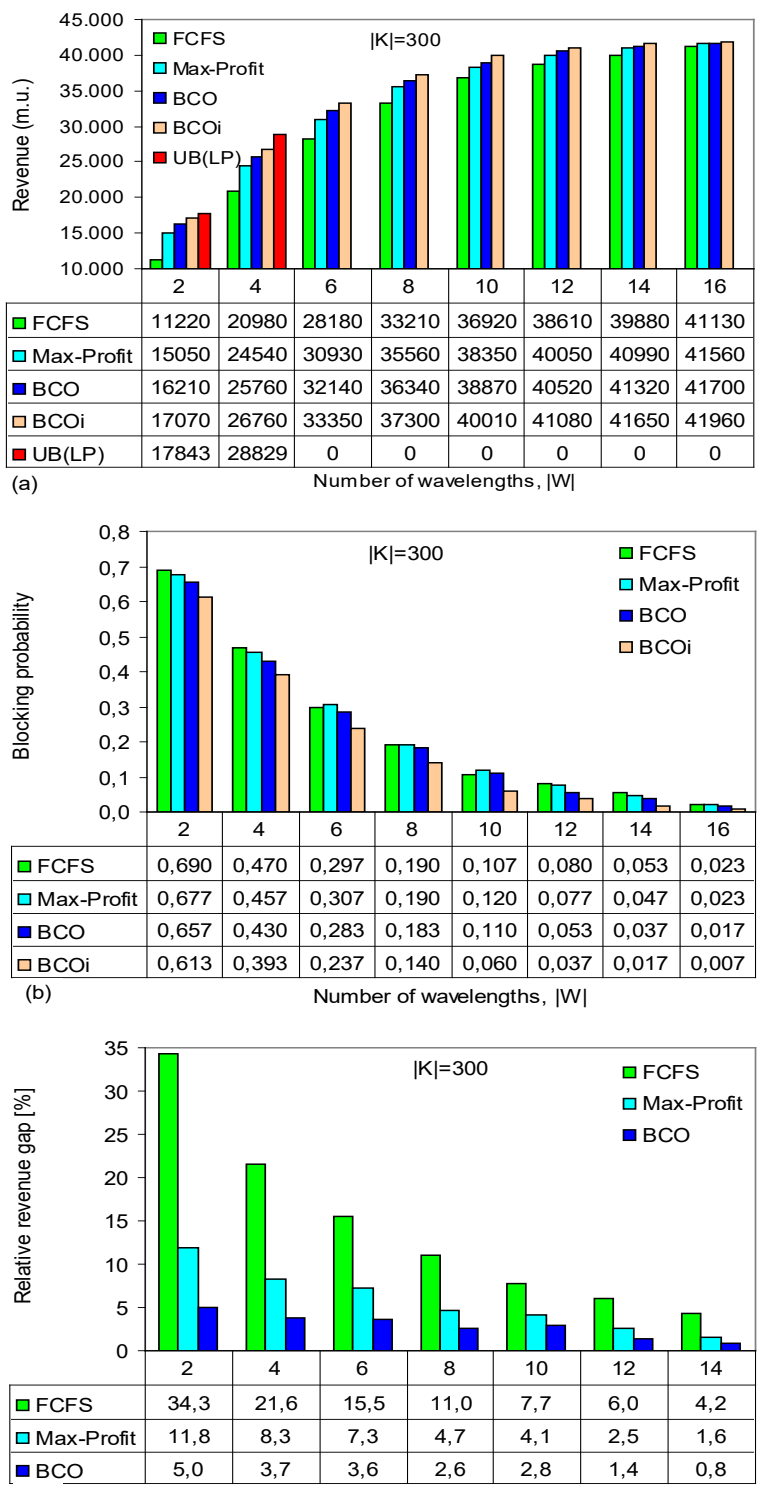

(c)

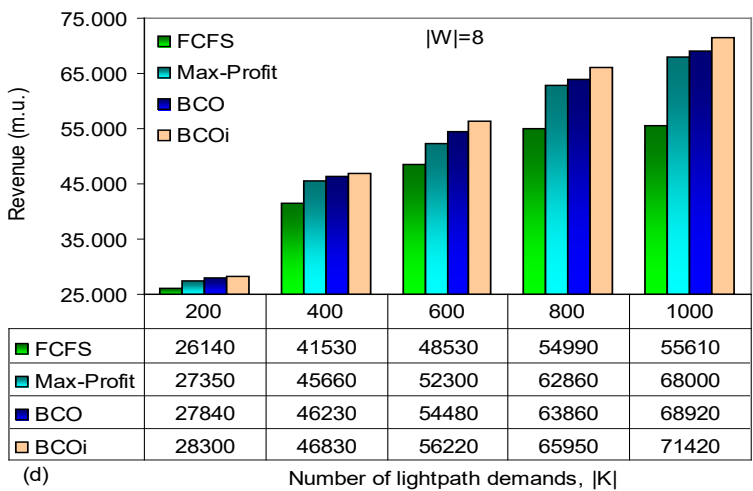

Fig. 6. Performance comparison for EON topology: (a) revenue vs. number of wavelengths, (b) number of rejected demands, (c) relative revenue gap, (d) revenue vs. number of demands
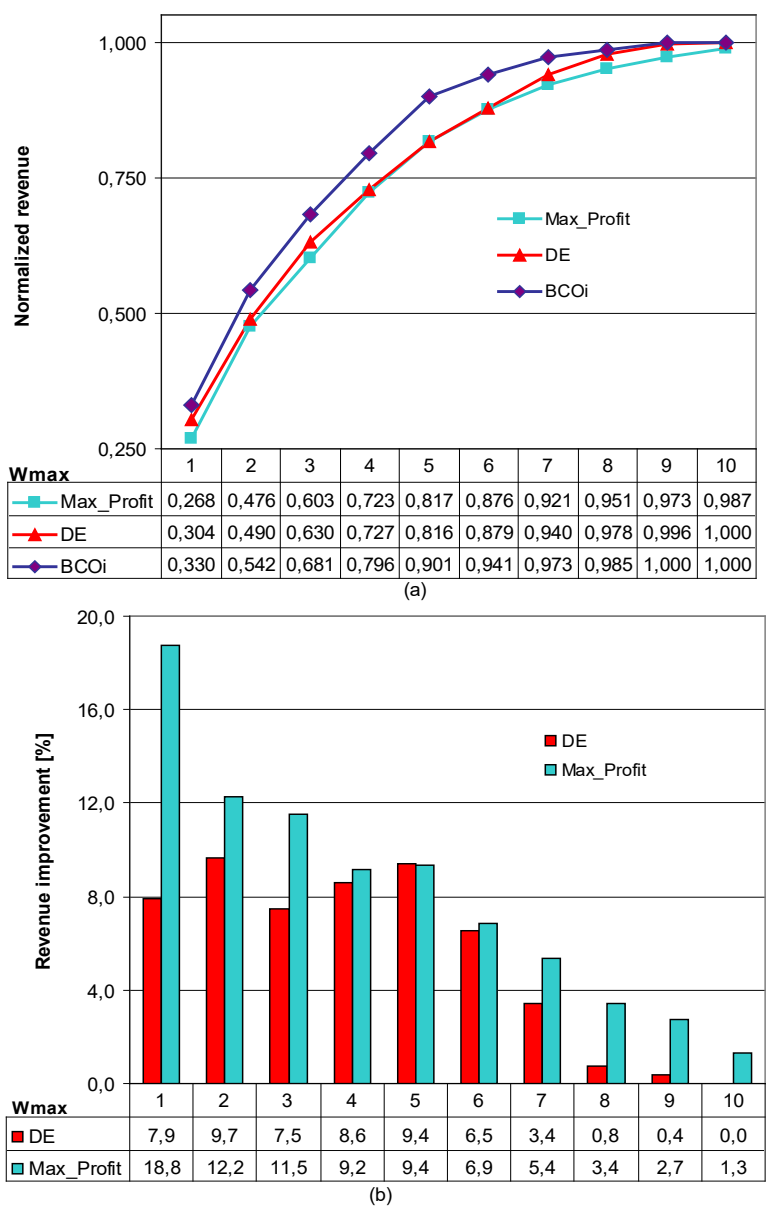

Fig. 7. Performance comparison for USA network topology with $|K|=100$ demands: a) normalized revenue, b) BCOi revenue improvement relative to $\mathrm{DE}$ and Max-Profit algorithm

Finally, we compare our BCOi approach to DE metaheuristic by performing simulation experiments in a more complex network topology, such as the USA network given by Fig 3.d. The control parameters of $\mathrm{DE}$, the mutation constant $(M)$ and the recombination constant $(R C)$ are defined by the user from the range $[0,1]$. As suggested in Refs.17-18 for this network topology, we used the following values of the mutation and the recombination constants: $M=0.2$ and $R C=0.5$, respectively. To make more fair comparison with our BCOi approach, we have used the number of individuals in DE population of $N P=10$ and $N P=20$, the same as the number of bees in BCOi algorithm. We have carried out the simulations in this network topology for two traffic scenarios: a) case of light traffic load with $|K|=100$ node pair demands and $b$ ) case of moderate-to-high traffic load with $|K|=500$ lightpath demands. Fig. 7 and Fig. 8 illustrate the comparison results for two considered traffic scenarios in USA network topology, respectively. 

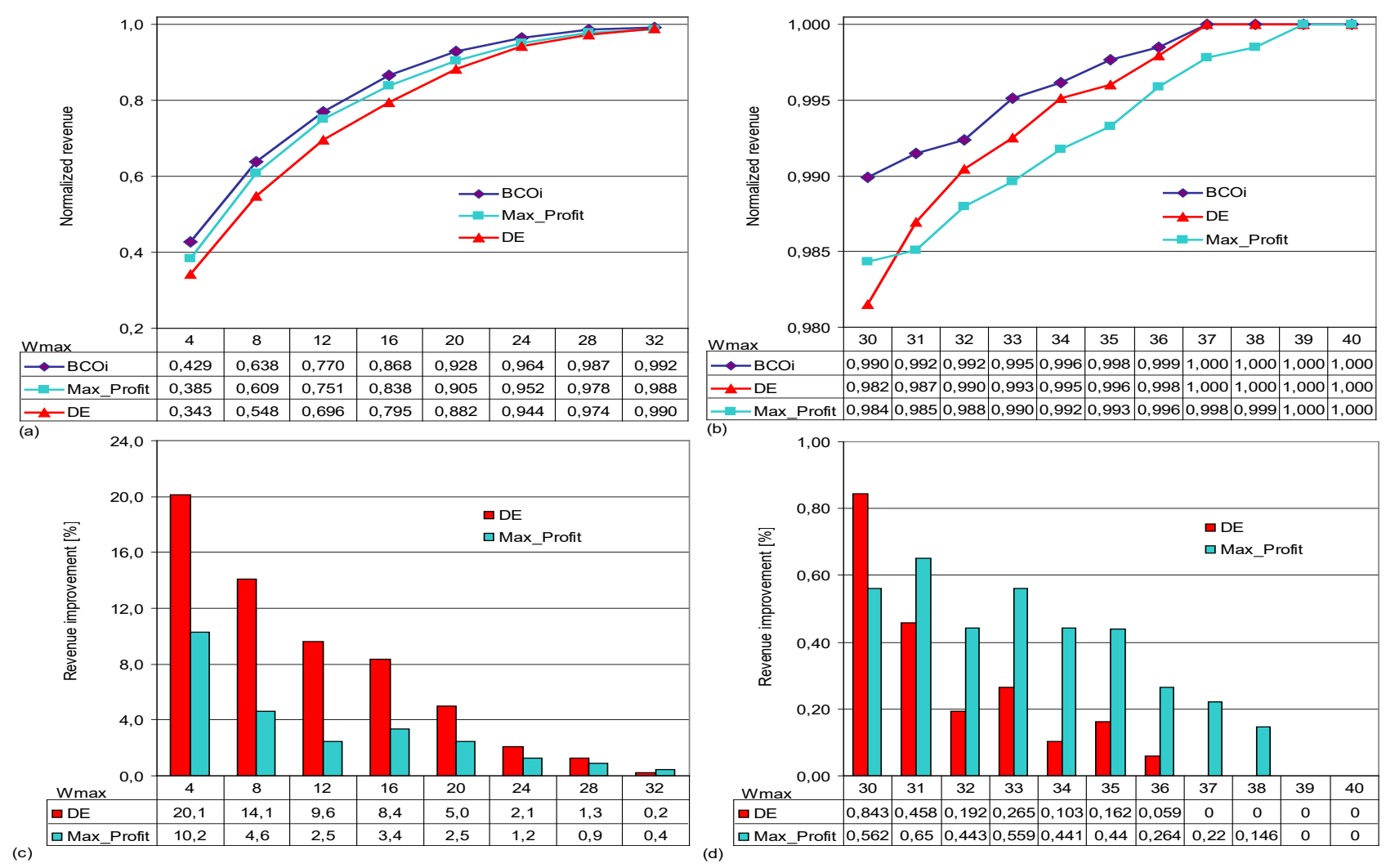

Fig 8. Performance comparison for USA network topology with $|\mathrm{K}|=500$ traffic demands: a) Normalized revenue value for scarce number of wavelengths, b) Normalized revenue value for more appropriate number of wavelengths, c) revenue improvement relative to DE and Max-Profit algorithm in the case of scarce number of wavelengths and d) revenue improvement relative to DE and MaxProfit algorithm in the case of more appropriate number of wavelengths.

Fig 7.a shows the comparison results of the reached revenue values with $\mathrm{BCO}, \mathrm{DE}$ and Max-Profit algorithms for different number of available wavelengths $\left(W_{\max }\right)$. The values are normalized to the maximal potential revenue value (i.e. the revenue that could be achieved if all the requested lightpaths could be established). It could be noticed that our BCOi algorithm almost always (for different values of $W_{\max }$ ) outperforms DE as well as Max-Profit algorithms. At the same time, DE produces revenues that are just slightly better than the Max-Profit heuristic. We can also see that for maximal normalized revenue value (1.0), the BCOi algorithm requires $W_{\max }=9$ wavelengths, while DE requires one more wavelength to provide the same revenue. Fig. 7.b shows the revenue improvement that could be achieved by our BCOi algorithm compared to DE and Max-profit algorithms. Similar results are obtained in the case of moderate-tolarge traffic scenario with $|K|=500$ demands (Fig. 8). However, to better perceive the performances of the considered algorithms, we divide the results into 2 scenarios: Figures 8.a and 8.c (on the left side) show the results in the case of scarce number of wavelengths, while Figures 8.b and 8.d (on the right side) refer to the case of more appropriately chosen number of wavelengths to the given traffic demands. As it can be seen from Figures 8.a and 8.b, our BCOi algorithm is once more superior to DE as well as to Max-Prof algorithms. However, it could be also noticed from Fig 8. a that in the case of scarce available wavelengths DE produces even worse results than Max-profit heuristic. The reason behind that could be found in a large number of demands $(|K|=500)$ and lack of useful information about the revenue values of individual lightpaths. With scarce available wavelengths and high number of demands, it is a challenge for DE to find a high quality complete RWA solution due to large possible random permutations while searching the solution space. By increasing the number of wavelengths, differences between the considered algorithms are reduced due to greater potential to establish more lightpaths (and consequently increase the revenues) even by using a simple heuristic, such as the Max-Profit algorithm. As a result, the relative 
revenue improvements of $\mathrm{BCO}$ algorithm are also reduced with increasing the number of wavelengths (Fig.8c).

If the available number of wavelengths is well matched to the considered traffic demands (Fig 8.b and Fig. 8.d), DE performs better than Max-Profit algorithm. However, it is still of inferior quality compared to the BCOi algorithm. Although the relative revenue improvement of BCOi compared to $\mathrm{DE}$ and Max-Profit algorithms does not seem to be too high in this case (as it could be seen from Fig 8.d, it is usually less than $1 \%$ ), the absolute revenue gain could be still important due to high maximal potential profit value (large number of lightpath demands). Based on the presented simulation results, one can notice that our BCOi algorithm always provides better objective (revenue) values compared to both, DE and Max-Profit algorithms. It proves our statement that BCOi approach is superior compared to other heuristic as well as metaheuristic algorithms.

Table 3. Number of variables, constraints and required CPU times for different algorithms in considered WDM networks

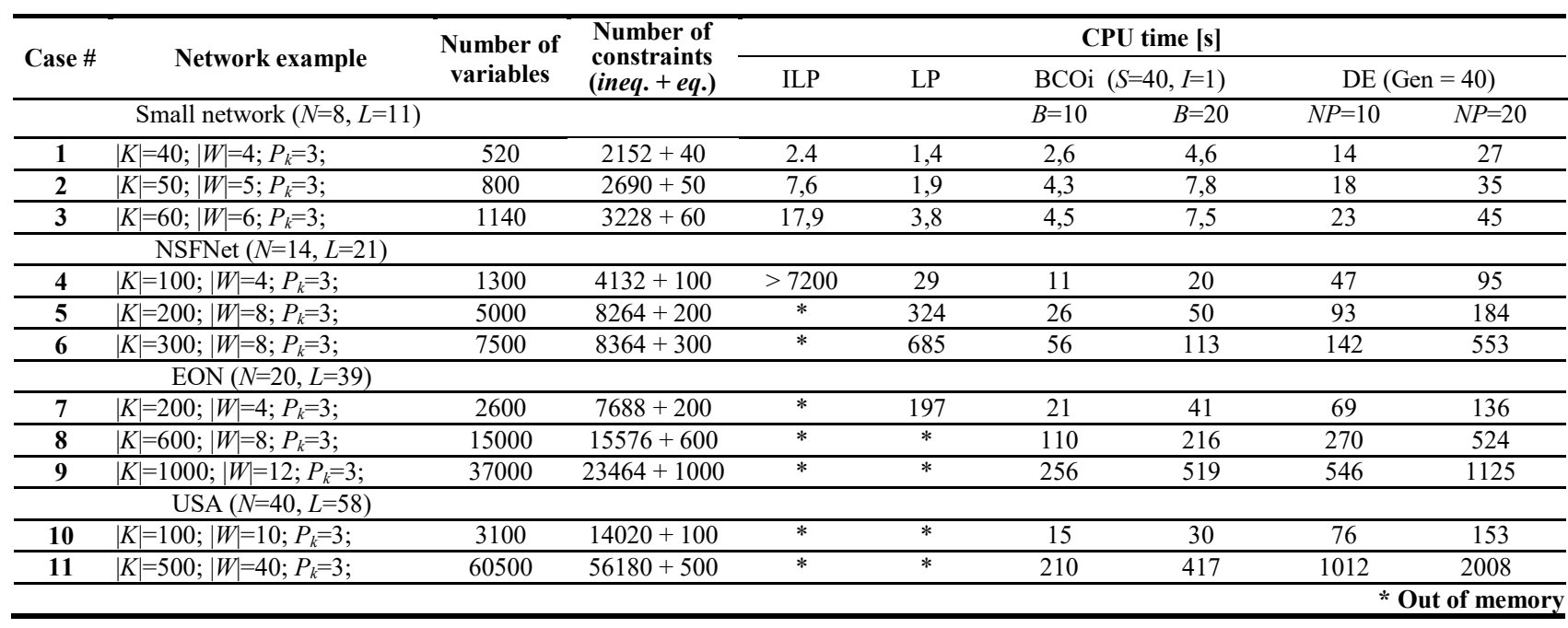

Finally, we analyse various approaches used in this study (ILP, LP relaxation, BCO and DE) according to the CPU times required to find the solution. Table 3 shows the summarised results for typical simulated scenarios in different WDM networks. As it could be noticed, the number of binary integer variables $(|K|(|P||W|+1))$ and the number of constraints: inequalities $(|W||L||T|+|K|)$ and equalities $(|K|)$ highly increase with problem dimensionality. Hence, the ILP and even LP-relaxation approaches become computationally intractable in case of larger problem dimensionality. It could be noticed that CPU times for BCOi approach does not increased rapidly with problem dimensionality, as in the case of ILP. Hence, our approach could be efficiently used even for large problem dimensionality and at the same time, it is able to produce high quality solutions. It has been also shown that such solutions could be obtained using a small population of bees (by experience we used $B=10$ bees in simulation experiments). However, by increasing the number of bees, the required CPU times could increase notably, but the solution quality is not improved too much. Hence, the population of bees does not need to be too large in order to obtain a high quality solution with our BCOi approach. We also compared the required CPU times of DE approach. The total number of generations Gen in DE should be as high as possible to obtain the best solution. Usually, it is chosen to be in the range between 100 and 1000 depending on the problem dimension ${ }^{17}$. However, to reduce the CPU time, we limited the number of generations in all simulations to Gen=200. We also assumed the same number of individuals $N P$ in $\mathrm{DE}$ as the number of bees $B$ in BCOi. Although the CPU times of two approaches could not be directly compared (due to different concepts of BCOi iterations and DE generations), to make some reasonable comparison we assumed here an analogy between $S$ steps of one BCO iteration (number of solutions modifications) and the number of generations Gen in DE. Using such an analogy, the required CPU times are compared, as shown in Table 3.

\section{Conclusion}

In this paper, we have demonstrated the lightpaths provisioning procedure over optical WDM networks under static traffic demands, where the call admission control, routing and wavelength assignment of lightpaths is based on revenue maximization from the 
point of view of network operators. An optimization model based on BCOi metaheuristic has been developed to solve the Max-Rev problem. We tested our approach by performing a number of simulation experiments in different WDM network topologies. It has been proved that our BCOi Max-Rev model is able to produce near optimal solutions, which significantly outperforms some other heuristic and metaheuristic algorithms. The main advantage of the proposed model is that it is able to find high quality solutions within reasonable computational (CPU) time even in case of high complex problems. Simulation experiments show that the quality of solutions does not depend too much on the number of bees in population. It has been also shown that our BCOi model does not require "well" initial solution in order to find a high quality final solution. Altogether, superior solution qualities jointly with high efficiency of the proposed BCOi algorithm confirm that it is absolutely indispensable for NOs to maximize their revenues while establishing the lightpaths over realistic optical WDM networks.

\section{Acknowledgements}

This study is supported by the Serbian Ministry of Education, Science and Technological Development under the research grant TR32025.

\section{References}

1. R. Ramaswami, K. N. Sivarajan, and G. H. Sasaki, Optical networks - a practical perspective (Morgan Kaufmann Publishers, 2010).

2. V. Anand and C. Qiao, Maximizing Profit in Optical WDM Networks, in Mobile Computing - part III, Operations Research/Computer Science Interfaces Series 19 (Kluwer Academic Publishers, New York, 2002), pp. 119-137. doi: 10.1007/0-306-47602-9_6

3. S. W. Lee, A. Chen, M.C. Yuang, A Lagrangean relaxation based near-optimal algorithm for advance lightpath reservation in WDM networks, Photon Netw Commun 19(1) (2010) 103-109. doi 10.1007/s11107009-0215-9

4. J. Li, K. Li, L. Wang and H. Zhao, Maximizing Profits of Routing in WDM Networks, Journal of Combinatorial Optimization 10 (2) (2005) 99-111. doi: 10.1007/s10878-005-2263-0

5. G. Sun, H. Yu, V. Anand, L. Li and H. Di, Optimal provisioning for virtual network request in cloud-based data centers. Photon Netw Commun 24(2) (2012) 118131. doi: $10.1007 / \mathrm{s} 11107-012-0372-0$

6. M. Shalom, P. W. H. Wong and S. Zaks, Profit Maximization in Flex-Grid All-Optical Networks, in
Structural Information and Communication Complexity, Lecture Notes in Computer Science, 8179 (2013) 249. 260. doi :10.1007/978-3-319-03578-9_2

7. G. Marković, Optimization of resource usage in optical wavelength routing networks. $P h D$ thesis, University of Belgrade, Serbia (2007) (origin in Serbian).

8. G. Marković, D. Teodorović and V. AćimovićRaspopović, Routing and wavelength assignment in alloptical networks based on the bee colony optimization, AI Communications 20(4) (2007) 273-285.

9. G. Marković, V. Aćimović-Raspopović and V. Radojičić, A heuristic algorithm for lightpath scheduling in next-generation WDM optical networks, Photonic Network Communications 23(3) (2012) 272284. doi: 10.1007/s11107-011-0358-3

10. T. Davidović, D. Teodorović and M. Šelmić, Bee Colony Optimization Part I: The Algorithm Overview. Yugoslav Journal of Operations Research 25(1) (2015) 33-56. doi: 10.2298/YJOR131011017D

11. D. Teodorović, M. Šelmić and T. Davidović, Bee Colony Optimization Part II: The Application Survey. Yugoslav Journal of Operations Research 25(2) (2015) 185-219. doi: 10.2298/YJOR131029020T.

12. T. Davidović, D. Ramljak, M. Šelmić and D. Teodorović, Bee colony optimization for the $p$-center problem. Computers and Operations Research 38(10), (2011) 1367-1376. doi:10.1016/j.cor.2010.12.002

13. D. Teodorović, Swarm intelligence systems for transportation engineering: principles and applications, Transp. Res. Part C: Emerg. Technol. 16(6) (2008) 651-667 doi: 10.1016/j.trc.2008.03.002

14. P. Lučić and D. Teodorović, Computing with bees: attacking complex transportation engineering problems, International Journal on Artificial Intell. Tools 12(3) (2003) 375-394. doi: 10.1142/S0218213003001289

15. D. Teodorović, P. Lučić, G. Marković, and M. Dell' Orco, Bee Colony Optimization: Principles and Applications, 8th IEEE NEUREL (2006) 151-156.

16. Das, S., Suganthan, P.N., Differential evolution: a survey of the state-of-the-art. IEEE Trans. Evolut. Comput. 15(1) (2011) 4-31. doi: 10.1109/TEVC.2010.2059031

17. F. Lezama, G. Castañón, A-M. Sarmiento, Routing and wavelength assignment in all optical networks using differential evolution optimization, Photonic Networks Communication 26(2-3) (2013) 103-119. doi: 10.1007/s11107-013-0413-3

18. F. Lezama, G. Castañón, A.M. Sarmiento, I.B. Martins, Differential evolution optimization applied to the routing and spectrum allocation problem in flexgrid optical networks, Photon. Netw. Commun. 31(1) (2016) 129-146. doi: 10.1007/s11107-015-0558-3

19. F. Lezama, G. Castañón, A-M. Sarmiento, Differential evolution optimization applied to the wavelength converters placement problem in all optical networks, Computer Networks 56 (2012) 2262-2275. doi: 10.1016/j.comnet.2012.03.019

20. G. Marković, Wavelength Converters Placement in Optical Networks Using Bee Colony Optimization, Advances in Electrical and Computer Engineering, 16(1) (2016) 3-10. doi: 10.4316/AECE.2016.0100 